The Age-Dependent Association Between Vascular Risk Factors and Depressed Mood

Maria Blöchl, MSc $\mathrm{MSc}^{1,2}$, H. Lina Schaare, $\mathrm{PhD}^{3,4}$, Ute Kunzmann, $\mathrm{PhD}^{5}$, Steffen Nestler, $\mathrm{PhD}^{1}$

${ }^{1}$ Department of Psychology, University of Münster

${ }^{2}$ Department for Neurology, Max Planck Institute for Human Cognitive and Brain Sciences, Leipzig

${ }^{3}$ Otto Hahn Group Cognitive Neurogenetics, Max Planck Institute for Human Cognitive and Brain Sciences, Leipzig

${ }^{4}$ Institute of Neuroscience and Medicine (INM-7: Brain and Behaviour), Research Centre Jülich

${ }^{5}$ Department of Psychology, University of Leipzig

Word count (excl. abstract and references): 5,206

Word count (abstract): 245

Number of references: 57

Number of data elements: 4

Author Note

Correspondence concerning this paper should be addressed to Maria Blöchl, Department of Psychology, University of Münster, Fliednerstraße 21, 48149 Münster, Germany; Telephone: + 49 251 8334127; Email: mbloechl@uni-muenster.de. 


\begin{abstract}
Objectives: Cumulative burden of vascular risk factors (VRFs) has been linked to an increased risk of depressed mood. However, the role of age in this association is still unclear. Here, we investigated whether VRF burden is associated with levels and changes in depressed mood and whether these associations become stronger or weaker from mid- to later life.
\end{abstract}

Method: We used longitudinal data from 5,689 participants (52-89 years) of the English Longitudinal Study of Ageing (ELSA). A composite score incorporated the presence of five VRFs: hypertension, diabetes, smoking, obesity, and hypercholesterolemia. Second-order latent growth models were used to test whether levels and changes of depressed mood differed as a function of baseline VRF burden, and whether these associations were moderated by age.

Results: Baseline VRF burden showed a small association with higher levels of depressed mood (estimate $=0.076 ; 95 \%$ CI $[0.024,0.129], p=.005)$. This association varied with age, such that it was stronger in midlife compared to later life (estimate $=-0.007 ; 95 \% \mathrm{CI}[-0.012,-0.002], p=.017$ ). There was no evidence that VRF burden was associated with changes in depressed mood.

Discussion: Our findings suggest that VRF burden in midlife, but less so in later life, predicts individual differences in depressed mood. These findings are consistent with reports on the importance of midlife VRFs and support the idea that promotion of vascular health in this age group or earlier in life may be critical to maintain mental health across adulthood.

Keywords: depression; cardiovascular health; vascular depression hypothesis; aging; latent growth models 


\section{The Age-Dependent Association between Vascular Risk Factors and \\ Depressed Mood}

In the past two decades, modifiable vascular risk factors (VRFs), such as hypertension, obesity, smoking, diabetes, and hypercholesterolemia, have emerged as major targets to promote healthy aging (Lloyd-Jones et al., 2010). Numerous studies show that the presence of VRFs, in particular the cumulative burden from multiple risk factors in midlife, is associated with an increased risk of adverse health outcomes in later life (Lloyd-Jones et al., 2010; Qiu \& Fratiglioni, 2015; Stamler et al., 1999). However, how VRF burden relates to trajectories of depressed mood and whether these relations vary across adulthood is less clear. This issue is key as mental health is an important aspect of healthy aging (World Health Organisation, 2019) and to maintain mental health, the timing of public health interventions to improve vascular health may be important. In the current study, we examined the associations of VRF burden with levels and longitudinal changes of depressed mood, and whether these associations are dependent on age.

\section{Vascular Risk Factor Burden and Depressive Symptoms}

Considerable theoretical work has implicated VRF burden in the pathophysiology of depression, especially in later life (Alexopoulos, 1997; Aziz \& Steffens, 2013; Gaupp et al., 2000). One of the most prominent theories, the vascular depression hypothesis, postulates that VRFs "predispose, precipitate, or perpetuate" depressive symptoms in older adults (Alexopoulos, 1997). This link is thought to arise because VRFs contribute to small vessel damage in the brain, which can compromise the integrity of fronto-subcortical circuits involved in mood regulation (Smagula \& Aizenstein, 2016; Taylor et al., 2013). However, while some cross-sectional and longitudinal studies have supported the vascular depression hypothesis (Kivimäki et al., 2012; Luijendijk et al., 2008; Mast et al., 2005), other studies failed to demonstrate an association between VRFs and depressive symptoms (Lyness et al., 2000; Stewart et al., 2001; Valkanova \& Ebmeier, 2013). A major drawback of previous studies is that they have rarely taken a lifespan approach: Either they 
only focused on older adults or they did not consider that the association with depressive symptoms may critically depend on the age at which VRFs are assessed.

\section{The Role of Age}

Although few studies have explicitly investigated the role of chronological age, most prior research presumed that the association between VRF burden and depression strengthens across the adult lifespan. VRF burden and associated microvascular damage are typically thought to increase with age (Laina et al., 2018; Lloyd-Jones et al., 2010). In line with the vascular depression hypothesis, it has been hypothesized that mood disturbances may only emerge if vascular burden reaches a certain threshold, above which it compromises the ability to regulate emotions or cope with age-related declines (Alexopoulos, 1997; Smagula \& Aizenstein, 2016; Taylor et al., 2013). Direct evidence for this hypothesis is, however, limited. Only one cross-sectional study reported a significant association between VRF burden and depressive symptoms in individuals who were older but not younger than 85 years (Mast et al., 2005). Importantly, this finding can only be interpreted with caution, given that the authors' index of VRF burden included heart diseases. Manifest vascular diseases, such as heart diseases, are more common in later life and might cause depression not because of vascular-related pathology but because of their life-threatening nature and effects on functional abilities (Kivimäki et al., 2012; Lenze et al., 2001; Valkanova \& Ebmeier, 2013). Accordingly, manifest vascular diseases have been established as strong risk factor for depression (e.g. Wikman et al., 2011). The distinction between manifest vascular diseases and VRFs is critical, and it would be necessary to establish similar age-dependent effects in the absence of manifest vascular diseases.

Notably, several lines of research point to the alternative prediction, suggesting that VRF burden is a more powerful predictor of depressive symptoms in midlife compared to later life. First, psychological research has shown age-related gains in emotion regulatory abilities and motivational priorities, which arguably help adults to better adapt to losses in daily functioning 
and physical health as they age (Charles, 2010; Charles \& Carstensen, 2010). In line with this notion, mood and well-being are well-maintained until old age, despite an age-related increase in VRF burden (Almeida, 2008; Sutin et al., 2013). Moreover, an increase in health problems, such as a high VRF burden, can be seen as almost normative in later life (Barrett \& Gumber, 2020; Ubel et al., 2005; Wrosch \& Heckhausen, 2005). Middle-aged adults, for whom VRFs are less normative, may have more difficulties to psychologically adjust to the diagnosis and management of such risk factors (e.g., intake of medications) than older adults. Finally, accumulating research on physical and cognitive health taking a lifespan perspective suggests that the detrimental effect of VRF burden is particularly pronounced in midlife and tends to decrease with age (Pase et al., 2018; Qiu et al., 2005; Qiu \& Fratiglioni, 2015).

Preliminary evidence suggesting the association between VRF burden and depressive symptoms weakens with age exists but is still limited. More specifically, Kivimäki et al. (2012) found that in individuals younger than 65 years, but not in those older than 65 years, VRF burden - in the absence of manifest vascular disease - predicted depressive symptoms five years later. However, this study did not explicitly and systematically investigate the role of age at which VRFs were assessed. Moreover, it only focused on few follow-ups, which does not allow to draw firm conclusions about longitudinal changes in depressive symptoms. Studies with multiple follow-ups provide a crucial inroad into longitudinal changes in depressive symptoms. Importantly, they also allow to address whether VRFs are associated with faster mood declines over time, possibly indicative of slowly progressing damage to fronto-subcortical structures. Only few longitudinal studies have assessed depressive symptoms at multiple time points to test whether trajectories differ as a function of VRFs (Montagnier et al., 2014; Virtanen et al., 2017) and none have focused on cumulative burden of VRFs or investigated age-differential effects.

\section{The Present Study}

In the present study, we aimed to investigate the age-dependent relationship between VRF 
burden and trajectories of depressed mood. More specifically, we sought to examine the extent to which VRF burden is associated with levels and changes in depressed mood, and whether these associations increase or decrease with age. If VRF burden is associated with mood because of underlying brain damage, the association of VRF burden and levels of depressed mood would strengthen with age, and a higher number of VRFs might also predict accelerated changes in depressed mood. If, however, the effects of age-related improvement in emotional and motivational competencies prevail, the association of VRF burden and depressed mood would presumably weaken with age.

To test these competing predictions, we leveraged data from 5,689 individuals (52-89 years) of the English Longitudinal Study of Ageing (ELSA), who had extensive assessments of VRFs at baseline and were followed-up five times at two-year intervals, covering a period of ten years. We used second-order latent growth models (LGMs) to model trajectories of depressed mood over time, and test whether levels and changes differed as a function of baseline VRF burden, age, and their interaction. We focus on depressed mood since it lies at the heart of depression and is less susceptible to potential confounding effects of vascular health and age compared to somatic symptoms of depression (Bus et al., 2011; Schaakxs et al., 2017). Importantly, we only include participants who were free of manifest vascular diseases at baseline. Moreover, we statistically account for the presence of physical limitations, which are known to confound the association between VRF burden and depression (Almeida, 2008; Lyness et al., 2000; Valkanova \& Ebmeier, 2013), as well as for gender, level of education, and ethnicity.

\section{Methods}

\section{Sample}

The English Longitudinal Study of Ageing (ELSA) is a large, multidisciplinary longitudinal study of community-dwelling adults aged 50 or older (i.e. the core sample) and their partners, some of which were younger (Steptoe et al., 2013). Data collection started in 2002 (Wave 1), with 
a nationally representative sample being drawn from the Health Survey for England (HSE). For the purpose of this study, we used data from Wave $2(2004-2005)$ as baseline, as this was the first assessment that included self-reported information of VRFs (diagnosed hypertension, history of smoking) as well as laboratory assessments (blood pressure, fasting glucose, body mass index [BMI], and cholesterol levels) taken during a nurse visit. Participants have been re-examined every two years, with repeated measures of depressive symptoms until Wave 7 (2014 - 2015), spanning a total of ten years. Participants were excluded if they were not part of the core sample, if they reported a manifest vascular disease (stroke, heart attack, congestive heart failure) or neurodegenerative disorder (Parkinson's disease, dementia, Alzheimer's disease) at baseline, or if they did not provide data in the nurse visit. Finally, we excluded 1,146 people with missing data on covariates. Thus, the final sample included 5,689 participants (Figure 1).

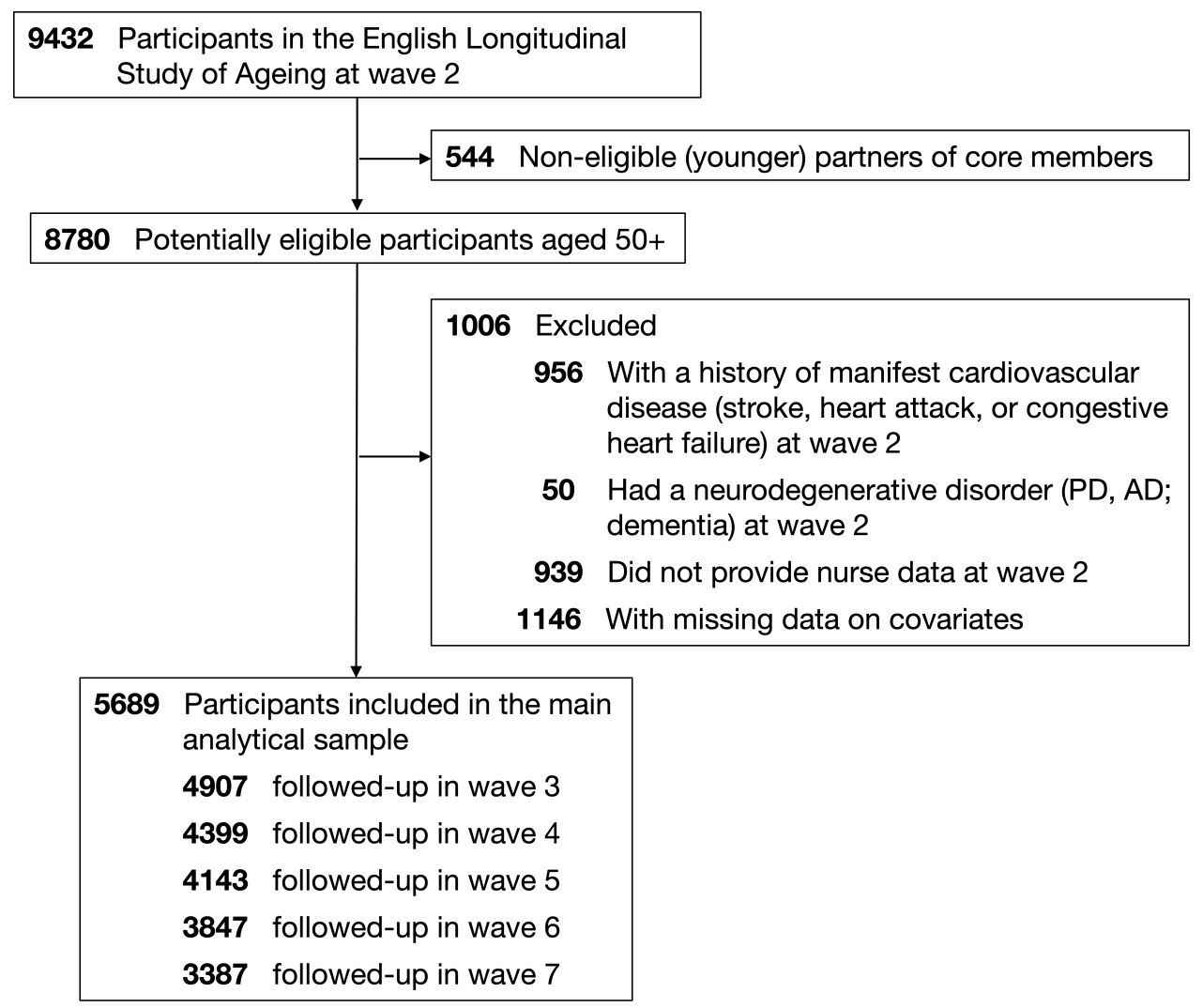

Figure 1. Flow chart of English Longitudinal Study of Ageing Sample participants included in the current study. 
As common in longitudinal studies of aging, sample attrition was sizable. Yet, $53 \%$ of the initial sample participated in all six waves of data collection and on average, participants contributed $4.64(S D=1.79$, Median $=6)$ waves of data. Attrition analyses indicated that those who contributed at five or six waves of survey data were younger $(d=0.54)$, had fewer physical limitations $(d=0.23)$, and were slightly more educated $(d=0.21)$ compared to those who contributed fewer than five waves of survey data. Differences in gender, ethnicity, depressive symptoms, and VRFs at baseline were negligible (all $d \mathrm{~s}<0.15$ ).

ELSA was conducted in accordance with the Declaration of Helsinki and informed consent was received from all participants to take part. Ethical approval and experimental protocols were granted by the Multicentre Research and Ethics Committee. The ELSA datasets can be accessed through the UK Data Service (https://www.ukdataservice.ac.uk).

\section{Measures}

\section{Depressed mood}

Depressed mood was measured using five affective items of the eight-item version of the Centre for Epidemiologic Studies Depression Scale (CES-D 8). The CES-D 8 is a well-established and widely used depression screening instrument for older adults (Turvey et al., 1999), in which participants are asked to report whether they had experienced eight symptoms 'much of the time' in the past week ('yes'/'no'). We focused on the five items tapping into depressed mood (i.e. felt depressed, was happy, felt lonely, enjoyed life, felt sad) because they reflect the core diagnostic criteria of major depressive disorder (MDD) and because somatic symptoms (three items: everything was an effort, sleep was restless, and could not get going) might inflate the observed associations with VRF burden during aging (Bus et al., 2011; Schaakxs et al., 2017).

\section{Vascular risk factor burden}


VRFs were assessed at baseline and included: hypertension, diabetes, current smoking, obesity, and hypercholesterolemia. Hypertension was defined as either reporting the presence of diagnosed hypertension or having systolic blood pressure $\geq 130 \mathrm{~mm} \mathrm{Hg}$ and diastolic blood pressure $\geq 80$ mm Hg (Muntner et al., 2019). Diabetes was defined as either reporting the presence of diagnosed diabetes or having a fasting blood glucose level of $\geq 7.0 \mathrm{mmol} / \mathrm{l}$ (Fox et al., 2015). Smoking was assessed using the core survey questionnaire by asking whether participants are currently smoking. Obesity was defined as a BMI of $\geq 30 \mathrm{~kg} / \mathrm{m}^{2}$ (Jensen et al., 2014). BMI was calculated from measurements of the participants' height and weight as assessed during the nurse visit. Hypercholesterolemia was defined as either reporting the presence of diagnosed hypercholesterolemia or high cholesterol or low-lipid density (LDL) cholesterol level of $\geq 4.1$ mmol/1 (Grundy et al., 2019).

Cumulative burden from these risk factors has been extensively linked to subsequent vascular disease, beyond the effect of single risk factors (Qiu \& Fratiglioni, 2015; Stamler et al., 1999). Thus, we calculated VRF burden as the number of individual risk factors. For this purpose, individual risk factors were coded as present (' 1 ') or absent (' 0 ') at baseline and then summed into an overall composite score (total score range 0 to 5 ).

\section{Age}

Information on participants' chronological age (in years) was provided by the ELSA team and computed from the difference between participants' date of birth and their date of the individual interview at baseline.

\section{Covariates}

Sociodemographic information included gender, educational attainment, and ethnicity. Educational attainment was defined as an indicator of having completed a higher or tertiary 
education ('yes'/'no'). Ethnicity was defined as a dichotomous variable ('white' / 'non-white'), since the vast majority of participants considered their ethnicity to be 'white'.

Physical limitations were included as a further covariate as they are known to increase the risk of depressed mood (Lenze et al., 2001; Sutin et al., 2013). Physical limitations were assessed by asking participants whether they experienced limitations in ten basic activities of daily living (ADLs; e.g. walking 100 yards, sitting about two hours, climbing one flight of stairs without resting, picking up a coin from a table). A scale ranging from 0 to 10 indicating the severity of physical limitations was computed (Spector \& Fleishman, 1998).

\section{Statistical Analyses}

We first fitted a series of longitudinal factor models to verify that stability and changes could be attributed to a common latent variable over time (Ferrer et al., 2008). We tested increasing levels of measurement invariance by successively introducing equality constraints on factor loadings, item thresholds, and factor variances in the measurement models of depressed mood, respectively. Strict measurement invariance constraints were satisfied, indicating that the same latent construct was being measured at each time of measurement (for more information on measurement invariance analyses and results, see Supplementary Results 1).

We then fitted an unconditional second-order latent growth models (LGMs; Hancock et al., 2001) to estimate the trajectory of depressed mood as a function of time. LGMs are structural equation models that allow for an examination of the initial level of an outcome (i.e. intercept), and its rate of change (i.e. slope), which describe the group-level growth curve of the outcome. Second-order LGMs additionally model the construct at each time point as a latent variable, which allows to model constructs free of measurement error, and to examine longitudinal measurement invariance. The unconditional second-order LGM fit the data well $\left(\chi^{2}(371)=1184.1, p<.001\right.$; $\mathrm{CFI}=.987 ; \mathrm{RMSEA}=0.018 ; \mathrm{SRMR}=.045)$, indicating a linear growth trajectory of depressed mood over time. 
Finally, our main model examined the extent to which growth trajectories between individuals differed as a function of baseline VRF burden, and how this effect varied by age. Conditional second-order LGMs were fitted that included VRF burden, age, and their interaction as predictors of between-person differences in levels (i.e. intercept) or changes (i.e. slope) in depressed mood (Supplementary Figure S1). We furthermore controlled for the effects of gender, education, ethnicity, and physical limitations. Age, VRF burden, and physical limitations were centered to their respective mean to aid the interpretation of the model parameters.

We fitted the statistical models using R (version 3.6.2) and the 'lavaan' package (Rosseel, 2012) (R Core Team, 2014) . For model estimation, we used a diagonally weighted least squares (DWLS) estimator with robust standard errors and a mean and variance adjusted test statistic. This estimator is denoted as means and variance adjusted weighted least square (WLSMV) in the 'lavaan' package. WLSMV accounts for the ordinal nature of the indicator variables and is generally robust to deviations from the assumption of multivariate normally distributed latent responses, especially when using relatively large samples (Flora \& Curran, 2004; Li, 2016; Rhemtulla et al., 2012). To handle missing data, we used a pairwise deletion approach.

Global model fit was evaluated based on three absolute fit indices: Comparative Fit Index (CFI; values $>0.95$ considered acceptable), Root Mean Square Error of Approximation (RMSEA; values $<0.06$ considered acceptable), and Standardized Root Mean Square Residual (SRMR; values $<0.08$ considered acceptable). To evaluate single parameters, we report point estimates and their $95 \%$ confidence interval (CI). For significance testing of parameter estimates, an alpha of .05 was used as a threshold, given that previous studies on vascular risk factors and depression tended to report small effects (Valkanova \& Ebmeier, 2013).

\section{Robustness Analyses}

Although pairwise deletion maximizes the available information when using WLSMV, we conducted several robustness analyses to rule out any potential biases due to missing data. First, 
we used data of our main analytic sample $(N=5,689)$ and re-fitted the final second-order LGM as a first-order LGM using the sum scores of depressed mood as manifest variables. Since sum scores can be regarded as metric variables, this approach allowed us to leverage robust Full Information Maximum Likelihood (robust FIML) estimation that assumes that the missing data is missing at random (MAR; Enders, 2001), which is a standard assumption in longitudinal studies with attrition. Second, we calculated another first-order LGM (with FIML) to account for potential biases due to missing covariate data. In this model, we additionally included the data of the 1,146 participants that were excluded from our main analysis due to lack of information on covariates.

A final analysis probed the robustness of our results when accounting for selective nonresponses. Although previous comparisons of sociodemographic characteristics suggested that the sample at Wave 1 was broadly representative of the general population (Steptoe et al., 2013), differential non-response at Wave 2 and our exclusion criteria might have led to a sample that is somewhat unrepresentative of the target population (i.e., persons born before 1 March 1952 living in private households in Britain). To account for selective non-responses in our models, we additionally considered Wave 2 sampling weights, which were provided by the ELSA team, when we fitted the first-order LGM (with FIML).

\section{Results}

\section{Sample Characteristics}

Sample characteristics are provided in Table 1 and zero-order correlations among all variables are presented in Supplementary Table S4. At baseline, the mean age of participants was 65.4 years $(S D=9.1$; range $=52-89)$ and 3,110 participants $(55 \%)$ were female. Depressed mood was generally low $(M=0.65, S D=1.19)$ but ranged across the full spectrum from zero to five, with 937 participants (17\%) reporting two or more symptoms. Overall VRF burden was moderate, with $1.46(S D=1.02)$ risk factors on average. 
Table 1

Participant Characteristics in the Overall Sample and Stratified by Age.

\begin{tabular}{lccccc}
\hline & & \multicolumn{4}{c}{ Age group } \\
\cline { 3 - 5 } & Whole sample & $50-59 \mathrm{y}$ & $60-69 \mathrm{y}$ & $70-79 \mathrm{y}$ & $>80 \mathrm{y}$ \\
\hline$n$ at baseline (\%) & $5,689(100)$ & $1,912(34)$ & $1,979(35)$ & $1,298(23)$ & $500(9)$ \\
Age in y, mean \pm s.d. & $65.36 \pm 9.08$ & $55.87 \pm 2.03$ & $64.38 \pm 2.94$ & $73.98 \pm 2.87$ & $83.20 \pm 2.42$ \\
Women, $n(\%)$ & $3,110(55)$ & $1,032(54)$ & $1,067(53)$ & $706(54)$ & $305(61)$ \\
White ethnicity, $n(\%)$ & $5,598(98)$ & $1,868(98)$ & $1,949(98)$ & $1,281(99)$ & $500(100)$ \\
No higher education, $n(\%)$ & $4,892(86)$ & $1,549(81)$ & $1,701(86)$ & $1,173(90)$ & $469(94)$ \\
ADL, mean \pm s.d. & $1.72 \pm 2.32$ & $1.30 \pm 2.18$ & $1.61 \pm 2.24$ & $2.05 \pm 2.34$ & $2.89 \pm 2.63$ \\
VRF burden, mean \pm s.d. & $1.46 \pm 1.02$ & $1.43 \pm 1.03$ & $1.48 \pm 1.03$ & $1.57 \pm 1.01$ & $1.22 \pm 0.92$ \\
Depressed mood, mean \pm s.d. ${ }^{\mathrm{b}}$ (\%) & & & & \\
$\quad$ Wave 2 (Baseline) & $0.65 \pm 1.19$ & $0.68 \pm 1.24$ & $0.54 \pm 1.11$ & $0.67 \pm 1.19$ & $0.93 \pm 1.29$ \\
Wave 3 & $0.58 \pm 1.17$ & $0.56 \pm 1.17$ & $0.51 \pm 1.11$ & $0.62 \pm 1.19$ & $0.89 \pm 1.27$ \\
Wave 4 & $0.58 \pm 1.14$ & $0.51 \pm 1.09$ & $0.57 \pm 1.15$ & $0.66 \pm 1.17$ & $0.82 \pm 1.19$ \\
Wave 5 & $0.63 \pm 1.19$ & $0.56 \pm 1.17$ & $0.57 \pm 1.12$ & $0.77 \pm 1.27$ & $0.99 \pm 1.31$ \\
Wave 6 & $0.57 \pm 1.13$ & $0.52 \pm 1.13$ & $0.51 \pm 1.06$ & $0.69 \pm 1.19$ & $0.94 \pm 1.36$ \\
Wave 7 & $0.53 \pm 1.11$ & $0.45 \pm 1.03$ & $0.49 \pm 1.06$ & $0.74 \pm 1.30$ & $0.95 \pm 1.41$ \\
\hline
\end{tabular}

Note: $\mathrm{ADL}=$ activities of daily living; $\mathrm{VRF}=$ vascular risk factor.

${ }^{\text {a }}$ Percentages do not exactly add up to $100 \%$ due to rounding.

${ }^{\mathrm{b}}$ A sum score was calculated from participants data for descriptive statistics, ranging from 0 to 5 (positive items were reverse coded). Scores represent the mean and SD across all participants' sum scores. 


\section{Age and Vascular Risk Factor Burden}

Table 1 shows the average VRF burden by 10-year age groups. The prevalence of baseline VRF burden increased across age groups and was highest in individuals aged 70-79 years (Mean= $1.57, S D=1.01$ ); however, VRF burden was again lower in the oldest age group, aged $\geq 80$ years $($ Mean $=1.22, S D=0.92)$. A breakpoint regression model confirmed a non-linear relationship between VRF burden and age: VRF burden increased slightly and linearly up to 75 years, and then dropped again (Supplementary Results 2).

\section{Associations between Vascular Risk Factor Burden and Levels of Depressed Mood}

Results of the second-order LGM revealed that VRF burden (estimate $=0.076 ; 95 \%$ CI $[0.024$, $0.129], p=.005)$, but not age (estimate $=-0.001 ; 95 \%$ CI $[-0.006,0.005], p=.939)$, showed a small association with levels of depressed mood (Table 2). Importantly, the association between VRF burden and levels of depressed mood was moderated by age. More specifically, this association was slightly weaker if VRFs were measured at an older age (estimate $=-0.007 ; 95 \%$ CI $[-0.012,-0.002], p=.017)$. Results depicted in Figure 2 illustrate that individuals with more risk factors reported more depressed mood at baseline, and this effect was stronger in midlife compared to later life. 
Table 2

Conditional Growth Curve Model of Depressed Mood Over Time $(N=5,689)$

\begin{tabular}{|c|c|c|c|c|c|c|}
\hline & \multicolumn{3}{|c|}{ Intercept } & \multicolumn{3}{|c|}{ Slope } \\
\hline & Est. & $95 \% \mathrm{CI}$ & Std. Est. & Est. & $95 \% \mathrm{CI}$ & Std. Est \\
\hline \multicolumn{7}{|l|}{ Fixed effects ${ }^{\mathrm{a}}$} \\
\hline & $-1.842 *$ & {$[-2.086,-1.597]$} & -1.386 & $-0.072 *$ & {$[-0.131,-0.021]$} & -0.304 \\
\hline Age & -0.001 & {$[-0.006,0.005]$} & -0.004 & $0.005^{*}$ & {$[0.003,0.007]$} & 0.188 \\
\hline Gender $^{\mathrm{b}}$ & $-0.431 *$ & {$[-0.548,-0.314]$} & -0.161 & 0.012 & {$[-0.023,0.048]$} & 0.026 \\
\hline Education $^{c}$ & -0.102 & {$[-0.252,0.049]$} & -0.027 & -0.016 & {$[-0.063,0.031]$} & -0.024 \\
\hline Ethnicity $^{\mathrm{d}}$ & $0.820^{*}$ & {$[0.397,1.243]$} & 0.077 & -0.076 & {$[-0.186,0.034]$} & -0.040 \\
\hline $\mathrm{ADL}$ & $0.170 *$ & {$[0.137,0.203]$} & 0.297 & 0.005 & {$[-0.004,0.014]$} & 0.048 \\
\hline VRF burden & $0.076^{*}$ & {$[0.024,0.129]$} & 0.059 & 0.004 & {$[-0.013,0.021]$} & 0.018 \\
\hline VRF burden $\times$ age & $-0.007 *$ & {$[-0.012,-0.002]$} & -0.045 & 0.001 & {$[-0.001,0.002]$} & 0.012 \\
\hline \multicolumn{7}{|l|}{ Random effects } \\
\hline Variance & $1.493 *$ & {$[0.954,2.032]$} & 0.845 & $0.053^{*}$ & {$[0.030,0.076]$} & 0.956 \\
\hline Covariance & -0.084 & {$[-0.168,0.001]$} & -0.298 & & & \\
\hline \multicolumn{7}{|l|}{ Model fit } \\
\hline$\chi^{2}(\mathrm{df})$ & \multicolumn{6}{|c|}{$621.66(567)$} \\
\hline CFI & \multicolumn{6}{|c|}{0.976} \\
\hline RMSEA & \multicolumn{6}{|c|}{0.004} \\
\hline SRMR & \multicolumn{6}{|c|}{0.050} \\
\hline
\end{tabular}

Note. $\mathrm{CI}=$ confidence interval; $\mathrm{ADL}=$ activities of daily living; $\mathrm{VRF}=$ vascular risk factor

a The fixed effect of the intercept in this model reflects the logit for answering the first items ('felt depressed') them with 'yes'; converting the estimates to probabilities (by $P\left(y_{1 i}=1\right)=1 / 1+\exp (-$ intercept $)$, indicates that the probability of answering the first with 'yes' is 0.14 , accounting for all covariates.

${ }^{\mathrm{b}}$ Reference category $=$ female

${ }^{\mathrm{c}}$ Reference category $=$ lower education

${ }^{\mathrm{d}}$ Reference category $=$ 'white'

$* p<.05$ 


\section{Associations between Vascular Risk Factor Burden and Changes of Depressed Mood}

Consistent with previous studies (e.g. Sutin et al., 2013), results of our second-order LGM furthermore revealed small decreases in depressed mood over time (estimate $=-0.072 ; 95 \%$ CI [0.131, -0.021], $p=.092$; see also Table 2 and Figure 2). This decrease was smaller for individuals who were older at baseline (estimate $=0.005 ; 95 \%$ CI $[0.003,0.007], p<.001)$. However, there was no evidence that VRF burden at baseline was associated with changes in depressed mood (estimate $=0.004 ; 95 \%$ CI $[-0.013,0.021], p=.653)$, or that this association was moderated by age $($ estimate $=0.001 ; 95 \%$ CI $[-0.001,0.002], p=.717)$ 


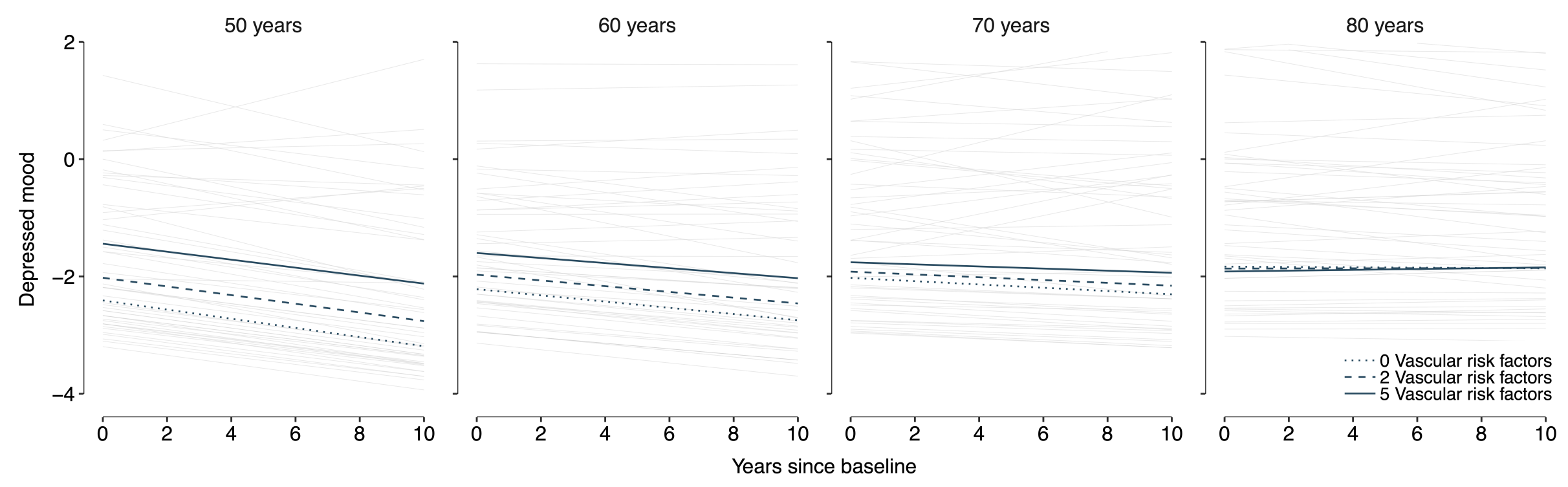

Figure 2. Trajectories of depressed mood over time as a function of baseline vascular risk factor (VRF) burden, age, and their interaction. The mean predicted trajectories for different levels of VRF burden are depicted in colour. Individual predicted trajectories are depicted in light grey. Scores for depressed mood can be converted to the probability of answering the first item of the depression questionnaire ('felt depressed') with 'yes'. Mean trajectories were plotted for a chosen profile of covariates (i.e., white females without a higher degree and mean ADLs). 


\section{Robustness Analyses}

Findings remained stable when we fitted a first-order LGM with a robust FIML estimator using sum scores of depressed mood as manifest indicators. Higher VRF burden was associated with higher levels of depressed mood (estimate $=0.040 ; 95 \%$ CI $[0.012,0.068], p=.006)$, and this association weakened with age (estimate $=-0.004 ; 95 \% \mathrm{CI}[-0.007,-0.001], p=.037$; see Table S5 in the Supplementary Materials for full results). Findings also remained stable, but were slightly attenuated, when we additionally included 1,146 participants with missing data on covariates $(N=$ 6,835). Higher VRF burden was associated with higher levels of depressed mood (estimate $=$ $0.027 ; 95 \%$ CI $[0.001,0.053], p=.048)$, and this association weakened with age (estimate $=$ $0.003 ; 95 \%$ CI $[-0.006,-0.001], p=.033$; see Table S6 in the Supplementary Materials for full results). Finally, findings also remained robust when employing sampling weights to account for selective non-response and sampling bias. Higher VRF burden was associated with higher levels of depressed $\operatorname{mood}($ estimate $=0.043 ; 95 \%$ CI [0.014, 0.073], $p=.003)$, and this association weakened with age (estimate $=-0.004 ; 95 \%$ CI $[-0.007,-0.001], p=.036$; see Table S7 in the Supplementary Materials for full results).

\section{Discussion}

This study examined how VRF burden relates to levels and changes of depressed mood and whether these associations vary with age. Our analyses revealed that a higher number of VRFs at baseline was associated with elevated levels of depressed mood, and that this association was moderated by age, such that it was stronger in middle-aged, as compared to older, individuals. However, these effects were small when accounting for sociodemographic factors and physical limitations, and there was no evidence that baseline VRF burden was associated with changes in depressed mood over subsequent years.

Age, Vascular Risk Factor Burden, and Levels of Depressed Mood 
VRFs have long been implicated in the development of depression, while empirical evidence for this hypothesis has been mixed (Alexopoulos, 1997; Aziz \& Steffens, 2013; Taylor et al., 2013; Valkanova \& Ebmeier, 2013). Here we found that the presence of multiple VRFs shows a small association with depressed mood, a core feature of depression, but that this association critically depends on the age window in which VRFs are assessed. This result could help to explain conflicting findings in previous research. More specifically, it is possible that there are differences in the age ranges of previous studies' samples and that studies with mainly middleaged or younger adults found stronger associations than studies that included mainly older adults. In addition, and similar to previous studies, our results suggest that after accounting for vascular diseases and physical limitations, there is limited evidence for an association between VRF burden and depressive symptoms in older adults (Kivimäki et al., 2012; Lyness et al., 2000; Valkanova \& Ebmeier, 2013).

Our results strengthen and extend findings from one previous study reporting preliminary evidence for age-dependent associations in depression (Kivimäki et al., 2012) by systematically investigating the moderating role of age in the association between VRF burden and depressed mood in individuals across a large age span (52 - 89 years). The current results are also in line with other studies showing the importance of midlife VRF burden for predicting vascular diseases, dementia and mortality (Pase et al., 2018; Qiu et al., 2005; Qiu \& Fratiglioni, 2015).

Notably, our results contradict findings from a cross-sectional study by Mast et al. (2005), who reported that unique associations between VRF burden and depression, beyond physical limitations, emerged only in later life. These divergent findings may be explained by differences in study design. Most importantly, Mast et al. used a risk index that incorporated the presence of heart diseases, which may have confounded the association with depression, especially in older adults. In contrast, people with vascular diseases were excluded in the present study. This approach allowed us to disentangle the effect of VRF burden and manifest vascular diseases, 
although we note that it may also have selected slightly healthier-than-average older participants. The results of our robustness analyses, however, suggest that this is unlikely to explain our results. More generally, our findings challenge predictions from the vascular depression hypothesis. While the vascular depression hypothesis has posited links between VRFs and depression in later life (Alexopoulos, 1997; Taylor et al., 2013), we show that these associations are already present and even stronger earlier in life. Our findings therefore underline the importance of taking a lifespan perspective to further elucidate the association between vascular health and depression and clarify the mechanisms underlying this association. They furthermore bear potential relevance for public health, as they suggest that preventing the accumulation of VRFs in midlife or even earlier, rather than in later life, may be important to maintain mental health.

Why precisely the association between VRF burden and levels of depressed mood weakens with age remains an open question for future research. It is possible that in later life, a decline in VRF burden is associated with looming pathological processes and general health deterioration (Qiu et al., 2005). These processes may lead to general increases in levels of depressed mood, which we also observed in our study, and weaken the association between VRFs and depressed mood in older age.

It is also plausible that psychological mechanisms explain the current pattern of results. Specifically, an increasing body of evidence suggests that older adults place a greater emphasis on emotion regulation strategies that allow for the preservation of well-being and reduction of stress (Charles, 2010; Charles \& Carstensen, 2010). Such age-related benefits may also protect older adults' mood in the presence of VRFs. For example, when faced with diabetes, recent studies showed that older adults report fewer diabetes-related stressors (Berg et al., 2020) or experience such stressors as less negative (Helgeson et al., 2020) compared to younger adults. Therein, expectations about aging may also play a role. The presence of some VRFs may be considered normative in later life, as it is more in keeping with the expected patterns of aging. Moreover, 
people tend to compare themselves to their same-age peers when asked to rate their physical health (Jylhä, 2009; Ubel et al., 2005). This comparison may be more detrimental for middle-aged adults with multiple VRFs, whose peers are generally healthier, than for older adults, whose peers tend to have a higher number and more severe health problems. Alternatively, medical care and treatment required by some risk factors may influence age- and health-related self-perceptions, and in turn take a stronger toll on mental health in middle-aged adults than in older adults (Barrett \& Gumber, 2020).

A final possibility refers to personality changes with age. More specifically, age-related decreases in neuroticism (e.g., Steunenberg et al., 2005) might explain why the association between VRF burden and depressed mood weakens with age. As individuals become more emotionally stable, they might worry less about their health and report less depressive symptoms (Lahey, 2009). Decreases in neuroticism may facilitate age-related improvement in emotion regulation, which could help older individuals to cope with VRFs. Future research is needed to test the relative or unique contributions of different psychological mechanisms underlying the association between VRF burden and depressive symptoms. To disambiguate different hypotheses, it would be important to assess potential mediating and predictive factors (e.g., emotion regulation, neuroticism, daily stress processes, self-perceptions of health and aging, and treatment information) separately and ideally on multiple levels (self- and other-reports, performance-based).

\section{Age, Vascular Risk Factor Burden, and Changes of Depressed Mood}

Our analyses furthermore showed no evidence that changes in depressed mood over time varied as a function of baseline VRF burden, neither in midlife nor later life. Importantly, this suggests that the link between VRF burden in midlife and subsequent depression observed in previous longitudinal studies (Kivimäki et al., 2012) may reflect the persistence of earlier differences, rather than differential rates of age-related mood decline. This result also calls into question 
previously hypothesized mediating pathways. If midlife VRF burden relates to depression in later life because of vascular pathology or progressing brain damage, as frequently hypothesized (Smagula \& Aizenstein, 2016; Taylor et al., 2013), we arguably should have observed that VRF burden predicts age-related mood declines over time.

However, we acknowledge that the lack of associations with changes in depressed mood could be due to limitations of our study design. More specifically, the present sample did not include information on VRF burden in earlier adulthood. It is possible that the adverse effects of VRF burden on brain structure and function were already present in at least some middle-aged individuals before the study begun, especially if they had suffered from VRFs for some time (Maillard et al., 2012; Schaare et al., 2019). Future studies should investigate this possibility, for example, by recruiting younger adults and taking the time of onset of VRFs into account.

\section{Limitations and Future Directions}

The present study had several strengths, including the longitudinal approach with a focus on trajectories of depressed mood using second-order LGMs, the use of a large, age-heterogeneous sample, and the exclusion of individuals with manifest vascular diseases. These strengths need to be put in the context of some limitations. First, the design of the study is not ideally suited to make firm conclusions about the causality and directionality between VRF burden and depressed mood. For example, there is also evidence that depression in earlier life can promote the development of VRFs (Matthews et al., 2019). Repeated measurements of VRF burden would allow for a stronger design allowing to estimate time-ordered, reciprocal effects and to clarify potential bidirectional relationships across the lifespan. Alternatively, randomized controlled trials could test whether targeted public health efforts and prevention of VRFs in midlife, or earlier, will ultimately reduce depressive symptoms across adulthood. Second, our sample only included people from the fifth decade of their life onward. In the light of our results and given that VRFs typically develop earlier in life (Karmali \& Lloyd-Jones, 2014; Lloyd-Jones et al., 2010), more research including 
young and young middle-aged adults is needed to elucidate when precisely the observed associations arise. Third, we operationalized some VRFs by combining self-reported clinical diagnosis with laboratory assessments. While this approach improves the correct classification of people with VRFs such as hypertension or hypercholesterolemia, it does not allow to distinguish between the undetected presence, diagnosis, and treatment of risk factors. How much of the observed effects might be attributable to changes in self-views due to a diagnosis or perhaps side effects of medications require further research. Finally, generalizations of our findings are furthermore constrained by a rather homogeneous sample with respect to ethnicity. Future studies extending our findings to more diverse samples are therefore warranted.

\section{Conclusion}

Cumulative burden of VRFs in midlife appears to be a stronger predictor of levels of depressed mood compared to VRF burden in later life. This suggests that taking a lifespan perspective and focusing on age spans prior to reaching old age may be critical to understand the associations between risk factors for vascular diseases and depressed mood. As midlife is an important period in life to pave the way for healthy aging, preventing and managing VRFs in middle-aged or younger adults, rather than older adults, may be important to maintain mental health.

\section{Funding}

Financial support for the English Longitudinal Study of Ageing (ELSA) was provided by the National Institute on Aging (Grant RO1AG7644) and by a consortium of UK government departments coordinated by the UK Economic and Social Research Council. The funders had no role in the study design, data collection, data analysis, data interpretation, or writing of this manuscript.

\section{Acknowledgements}


The authors are grateful for the immense effort of the ELSA team as well as their participants for collecting and providing the original data. As such, the investigators within the ELSA contributed to the design and implementation of but did not participate in analysis or writing of this manuscript.

\section{Transparency Statement}

All R scripts used in the analysis and accompanying information to reproduce the results are available at https://osf.io/epvk4/?view_only=b5dee6c8272f4140b1a373282b51bdbe. The data is open access upon registration at https://www.ukdataservice.ac.uk. More information on the materials used in ELSA can be found at https://www.elsa-project.ac.uk. This study was not preregistered.

\section{References}

Alexopoulos, G. S. (1997). "Vascular depression” hypothesis. Archives of General Psychiatry, 54(10), 915-922. https://doi.org/10.1001/archpsyc.1997.01830220033006

Almeida, O. P. (2008). Vascular depression: Myth or reality? International Psychogeriatrics, 20(4), 645-652. https://doi.org/10.1017/S1041610207006473

Aziz, R., \& Steffens, D. C. (2013). What are the causes of late-life depression? The Psychiatric Clinics of North America, 36(4), 497-516. https://doi.org/10.1016/j.psc.2013.08.001

Barrett, A. E., \& Gumber, C. (2020). Feeling old, body and soul: The effect of aging body reminders on rge identity. The Journals of Gerontology: Series B, 75(3), 625-629. https://doi.org/10.1093/geronb/gby085

Berg, C. A., Helgeson, V. S., Kelly, C. S., Tracy, E. L., Litchman, M. L., \& Butner, J. E. (2020). Age differences in reactivity to daily general and Type 1 diabetes stressors. Psychology and Aging. https://doi.org/10.1037/pag0000519 
Bus, B. a. A., Marijnissen, R. M., Holewijn, S., Franke, B., Purandare, N., de Graaf, J., den Heijer, M., Buitelaar, J. K., \& Voshaar, R. C. O. (2011). Depressive symptom clusters are differentially associated with atherosclerotic disease. Psychological Medicine, 41(7), 1419-1428. https://doi.org/10.1017/S0033291710002151

Charles, S. T. (2010). Strength and vulnerability integration: A model of emotional well-being across adulthood. Psychological Bulletin, 136(6), 1068-1091. https://doi.org/10.1037/a0021232

Charles, S. T., \& Carstensen, L. L. (2010). Social and emotional aging. Annual Review of Psychology, 61(1), 383-409. https://doi.org/10.1146/annurev.psych.093008.100448

Downer, B., Estus, S., Katsumata, Y., \& Fardo, D. W. (2014). Longitudinal trajectories of cholesterol from midlife through late life according to Apolipoprotein E allele status. International Journal of Environmental Research and Public Health, 11(10), 10663-10693. https://doi.org/10.3390/ijerph111010663

Enders, C. K. (2001). The performance of the Full Information Maximum Likelihood estimator in multiple regression models with missing data. Educational and Psychological Measurement, 61(5), 713-740. https://doi.org/10.1177/0013164401615001

Ferrer, E., Balluerka, N., \& Widaman, K. F. (2008). Factorial invariance and the specification of second-order latent growth models. Methodology: European Journal of Research Methods for the Behavioral \& Social Sciences, 4(1), 22-36. https://doi.org/10.1027/16142241.4.1.22

Flora, D. B., \& Curran, P. J. (2004). An empirical evaluation of alternative methods of estimation for confirmatory factor analysis with ordinal data. Psychological Methods, 9(4), 466-491. https://doi.org/10.1037/1082-989X.9.4.466

Fox, C. S., Golden, S. H., Anderson, C., Bray, G. A., Burke, L. E., de Boer, I. H., Deedwania, P., Eckel, R. H., Ershow, A. G., Fradkin, J., Inzucchi, S. E., Kosiborod, M., Nelson, R. G., 
Patel, M. J., Pignone, M., Quinn, L., Schauer, P. R., Selvin, E., \& Vafiadis, D. K. (2015). Update on prevention of cardiovascular disease in adults with Type 2 diabetes mellitus in light of recent evidence: A scientific statement from the American Heart Association and the American Diabetes Association. Diabetes Care, 38(9), 1777-1803. https://doi.org/10.2337/dci15-0012

Gaupp, R., Berrios, G. E., \& Pomarol-Clotet, E. (2000). Depressive states in old age. (Classic Text No. 42). History of Psychiatry, 11(42 Pt 2), 213-225. https://doi.org/10.1177/0957154X0001104205

Grundy, S. M., Stone, N. J., Bailey, A. L., Craig, B., Birtcher, K. K., Blumenthal, R. S., Braun, L. T., Sarah, de F., Joseph, F.-T., Forman, D. E., Ronald, G., Heidenreich, P. A., Hlatky, M. A., Jones, D. W., Donald, L.-J., Nuria, L.-P., Ndumele, C. E., Orringer, C. E., Peralta, C. A., ... Joseph, Y. (2019). Guideline on the management of blood cholesterol: Executive summary: A report of the American College of Cardiology/American Heart Association task force on clinical practice guidelines. Circulation, 139(25), e1046-e1081. https://doi.org/10.1161/CIR.0000000000000624

Hancock, G. R., Kuo, W.-L., \& Lawrence, F. R. (2001). An illustration of second-order latent growth models. Structural Equation Modeling: A Multidisciplinary Journal, 8(3), 470-489. https://doi.org/10.1207/S15328007SEM0803_7

Helgeson, V. S., Van Vleet, M., \& Zajdel, M. (2020). Diabetes stress and health: Is aging a strength or a vulnerability? Journal of Behavioral Medicine, 43(3), 426-436. https://doi.org/10.1007/s10865-019-00106-4

Jensen, M. D., Ryan, D. H., Ard, J. D., Donato, K. A., Hu, F. B., Hubbard, V. S., Jakicic, J. M., Kushner, R. F., Loria, C. M., Millen, B. E., Nonas, C. A., Xavier, P.-S. F., June, S., Stevens, V. J., Wadden, T. A., Wolfe, B. M., \& Yanovski, S. Z. (2014). 2013 AHA/ACC/TOS Guideline for the management of overweight and obesity in adults. 
Circulation, 129(25_suppl_2), S102-S138. https://doi.org/10.1161/01.cir.0000437739.71477.ee

Jylhä, M. (2009). What is self-rated health and why does it predict mortality? Towards a unified conceptual model. Social Science \& Medicine (1982), 69(3), 307-316. https://doi.org/10.1016/j.socscimed.2009.05.013

Karmali, K. N., \& Lloyd-Jones, D. M. (2014). Achieving and maintaining cardiovascular health across the lifespan. Current Epidemiology Reports, 1(2), 75-81. https://doi.org/10.1007/s40471-014-0011-7

Kivimäki, M., Shipley, M. J., Allan, C. L., Sexton, C. E., Jokela, M., Virtanen, M., Tiemeier, H., Ebmeier, K. P., \& Singh-Manoux, A. (2012). Vascular Risk Status as a Predictor of LaterLife Depressive Symptoms: A Cohort Study. Biological Psychiatry, 72(4), 324-330. https://doi.org/10.1016/j.biopsych.2012.02.005

Lahey, B. B. (2009). Public health significance of neuroticism. The American Psychologist, 64(4), 241-256. https://doi.org/10.1037/a0015309

Laina, A., Stellos, K., \& Stamatelopoulos, K. (2018). Vascular ageing: Underlying mechanisms and clinical implications. Experimental Gerontology, 109, 16-30. https://doi.org/10.1016/j.exger.2017.06.007

Lenze, E. J., Rogers, J. C., Martire, L. M., Mulsant, B. H., Rollman, B. L., Dew, M. A., Schulz, R., \& Reynolds, C. F. (2001). The association of late-life depression and anxiety with physical disability: A review of the literature and prospectus for future research. The American Journal of Geriatric Psychiatry, 9(2), 113-135. https://doi.org/10.1097/00019442-200105000-00004

Li, C.-H. (2016). Confirmatory factor analysis with ordinal data: Comparing robust maximum likelihood and diagonally weighted least squares. Behavior Research Methods, 48(3), 936949. https://doi.org/10.3758/s13428-015-0619-7 
Lloyd-Jones, L.-J., Hong, Y., Labarthe, D., Mozaffarian, D., Appel, L. J., Van Horn, L.,

Greenlund, K., Daniels, S., Nichol, G., Tomaselli, G. F., Arnett, D. K., Fonarow, G. C., Ho, M. P., Lauer, M. S., Masoudi, F. A., Robertson, R. M., Roger, V., Schwamm, L. H., Sorlie, P., ... Rosamond, W. D. (2010). Defining and setting national goals for cardiovascular health promotion and disease reduction. Circulation, 121(4), 586-613. https://doi.org/10.1161/CIRCULATIONAHA.109.192703

Luijendijk, H. J., Stricker, B. H., Hofman, A., Witteman, J. C. M., \& Tiemeier, H. (2008). Cerebrovascular risk factors and incident depression in community-dwelling elderly. Acta Psychiatrica Scandinavica, 118(2), 139-148. https://doi.org/10.1111/j.16000447.2008.01189.x

Lyness, J. M., King, D. A., Conwell, Y., Cox, C., \& Caine, E. D. (2000). Cerebrovascular risk factors and 1-year depression outcome in older primary care patients. American Journal of Psychiatry, 157(9), 1499-1501. https://doi.org/10.1176/appi.ajp.157.9.1499

Maillard, P., Seshadri, S., Beiser, A., Himali, J. J., Au, R., Fletcher, E., Carmichael, O., Wolf, P. A., \& DeCarli, C. (2012). Effects of systolic blood pressure on white-matter integrity in young adults in the Framingham Heart Study: A cross-sectional study. The Lancet Neurology, 11(12), 1039-1047. https://doi.org/10.1016/S1474-4422(12)70241-7

Mast, B. T., Azar, A. R., \& Murrell, S. A. (2005). The vascular depression hypothesis: The influence of age on the relationship between cerebrovascular risk factors and depressive symptoms in community dwelling elders. Aging \& Mental Health, 9(2), 146-152. https://doi.org/10.1080/13607860412331336832

Matthews, K. A., Jennings, J. R., Lee, L., \& Pardini, D. (2019). Depressive symptoms during childhood and cardiovascular risk factors in black and white men. Psychosomatic Medicine, 81(2), 176-183. https://doi.org/10.1097/PSY.0000000000000652 
Montagnier, D., Dartigues, J.-F., Rouillon, F., Pérès, K., Falissard, B., \& Onen, F. (2014). Ageing and trajectories of depressive symptoms in community-dwelling men and women: Ageing and trajectories of depressive symptoms. International Journal of Geriatric Psychiatry, 29(7), 720-729. https://doi.org/10.1002/gps.4054

Muntner, P., Shimbo, D., Carey, R. M., Charleston, J. B., Gaillard, T., Misra, S., Myers, M. G., Ogedegbe, G., Schwartz, J. E., Townsend, R. R., Urbina, E. M., Viera, A. J., White, W. B., \& Wright, J. T. (2019). Measurement of blood pressure in humans: A scientific statement from the American Heart Association. Hypertension (Dallas, Tex.: 1979), 73(5), e35-e66. https://doi.org/10.1161/HYP.0000000000000087

Pase, M. P., Davis-Plourde, K., Himali, J. J., Satizabal, C. L., Aparicio, H., Seshadri, S., Beiser, A. S., \& DeCarli, C. (2018). Vascular risk at younger ages most strongly associates with current and future brain volume. Neurology, 91(16), e1479-e1486.

https://doi.org/10.1212/WNL.0000000000006360

Qiu, C., \& Fratiglioni, L. (2015). A major role for cardiovascular burden in age-related cognitive decline. Nature Reviews Cardiology, 12(5), 267-277. https://doi.org/10.1038/nrcardio.2014.223

Qiu, C., Winblad, B., \& Fratiglioni, L. (2005). The age-dependent relation of blood pressure to cognitive function and dementia. The Lancet. Neurology, 4(8), 487-499. https://doi.org/10.1016/S1474-4422(05)70141-1

R Core Team. (2014). R: A language and environment for statistical computing. R Foundation for Statistical Computing. http://www.R-project.org/.

Rhemtulla, M., Brosseau-Liard, P. É., \& Savalei, V. (2012). When can categorical variables be treated as continuous? A comparison of robust continuous and categorical SEM estimation methods under suboptimal conditions. Psychological Methods, 17(3), 354-373. https://doi.org/10.1037/a0029315 
Rosseel, Y. (2012). lavaan: An R Package for Structural Equation Modeling. Journal of Statistical Software, 48(2), 1-36. https://doi.org/10.18637/jss.v048.i02

Schaakxs, R., Comijs, H. C., Lamers, F., Beekman, A. T. F., \& Penninx, B. W. J. H. (2017). Agerelated variability in the presentation of symptoms of major depressive disorder. Psychological Medicine, 47(03), 543-552. https://doi.org/10.1017/S0033291716002579

Schaare, H. L., Kharabian Masouleh, S., Beyer, F., Kumral, D., Uhlig, M., Reinelt, J. D., Reiter, A. M. F., Lampe, L., Babayan, A., Erbey, M., Roebbig, J., Schroeter, M. L., Okon-Singer, H., Müller, K., Mendes, N., Margulies, D. S., Witte, A. V., Gaebler, M., \& Villringer, A. (2019). Association of peripheral blood pressure with gray matter volume in 19- to 40year-old adults. Neurology, 92(8), e758-e773. https://doi.org/10.1212/WNL.0000000000006947

Smagula, S. F., \& Aizenstein, H. J. (2016). Brain structural connectivity in late-life Major Depressive Disorder. Biological Psychiatry: Cognitive Neuroscience and Neuroimaging, 1(3), 271-277. https://doi.org/10.1016/j.bpsc.2015.11.005

Spector, W. D., \& Fleishman, J. A. (1998). Combining activities of daily living with instrumental activities of daily living to measure functional disability. The Journals of Gerontology. Series B, Psychological Sciences and Social Sciences, 53(1), S46-57. https://doi.org/10.1093/geronb/53b.1.s46

Stamler, J., Stamler, R., Neaton, J. D., Wentworth, D., Daviglus, M. L., Garside, D., Dyer, A. R., Liu, K., \& Greenland, P. (1999). Low risk-factor profile and long-term cardiovascular and noncardiovascular mortality and life expectancy: Findings for 5 large cohorts of young adult and middle-aged men and women. JAMA, 282(21), 2012-2018. https://doi.org/10.1001/jama.282.21.2012 
Steptoe, A., Breeze, E., Banks, J., \& Nazroo, J. (2013). Cohort profile: The English Longitudinal Study of Ageing. International Journal of Epidemiology, 42(6), 1640-1648. https://doi.org/10.1093/ije/dys168

Steunenberg, B., Twisk, J. W. R., Beekman, A. T. F., Deeg, D. J. H., \& Kerkhof, A. J. F. M. (2005). Stability and change of neuroticism in aging. The Journals of Gerontology: Series B, 60(1), P27-P33. https://doi.org/10.1093/geronb/60.1.P27

Stewart, R., Prince, M., Richards, M., Brayne, C., \& Mann, A. (2001). Stroke, vascular risk factors and depression. British Journal of Psychiatry, 178(01), 23-28. https://doi.org/10.1192/bjp.178.1.23

Sutin, A. R., Terracciano, A., Milaneschi, Y., An, Y., Ferrucci, L., \& Zonderman, A. B. (2013). The trajectory of depressive symptoms across the adult life span. JAMA Psychiatry, 70(8), 803-811. https://doi.org/10.1001/jamapsychiatry.2013.193

Taylor, W. D., Aizenstein, H. J., \& Alexopoulos, G. S. (2013). The vascular depression hypothesis: Mechanisms linking vascular disease with depression. Molecular Psychiatry, 18(9), 963-974. https://doi.org/10.1038/mp.2013.20

Turvey, C. L., Wallace, R. B., \& Herzog, R. (1999). A revised CES-D measure of depressive symptoms and a DSM-based measure of major depressive episodes in the elderly. International Psychogeriatrics, 11(2), 139-148. https://doi.org/10.1017/S1041610299005694

Ubel, P. A., Jankovic, A., Smith, D., Langa, K. M., \& Fagerlin, A. (2005). What is perfect health to an 85-year-old?: Evidence for scale recalibration in subjective health ratings. Medical Care, 43(10), 1054-1057. https://doi.org/10.1097/01.mlr.0000178193.38413.70

Valkanova, V., \& Ebmeier, K. P. (2013). Vascular risk factors and depression in later life: A systematic review and meta-analysis. Biological Psychiatry, 73(5), 406-413. https://doi.org/10.1016/j.biopsych.2012.10.028 
Virtanen, M., Elovainio, M., Josefsson, K., Batty, G. D., Singh-Manoux, A., \& Kivimäki, M. (2017). Coronary heart disease and risk factors as predictors of trajectories of psychological distress from midlife to old age. Heart, 103(9), 659-665. https://doi.org/10.1136/heartjnl-2016-310207

Wikman, A., Wardle, J., \& Steptoe, A. (2011). Quality of life and affective well-being in middleaged and older people with chronic medical illnesses: A cross-sectional population based study. PLoS ONE, 6(4), e18952. https://doi.org/10.1371/journal.pone.0018952

Wills, A. K., Lawlor, D. A., Matthews, F. E., Sayer, A. A., Bakra, E., Ben-Shlomo, Y., Benzeval, M., Brunner, E., Cooper, R., Kivimaki, M., Kuh, D., Muniz-Terrera, G., \& Hardy, R. (2011). Life course trajectories of systolic blood pressure using longitudinal data from eight UK cohorts. PLOS Medicine, 8(6), e1000440. https://doi.org/10.1371/journal.pmed.1000440

World Health Organisation. (2019). 10 priorities. Towards a decade of healthy ageing. https://www.who.int/ageing/WHO-ALC-10-priorities.pdf

Wrosch, C., \& Heckhausen, J. (2005). Being on-time or off-time: Developmental deadlines for regulating one's own development. In Thinking time: A multidisciplinary perspective on time (pp. 110-123). Hogrefe \& Huber Publishers. 


\section{Supplementary Materials}

\section{The Age-Dependent Association Between Vascular Risk Factors and Depressed Mood}

Maria Blöchl, H. Lina Schaare, Ute Kunzmann, Steffen Nestler

Correspondence: mbloechl@uni-muenster.de

Supplementary materials include:

Illustration Growth Model (Figure S1) ............................................... p. p. 2

Measurement Invariance Analysis (incl. Tables S1 and S2) .................. p. $3 f$

Vascular Risk Factor Burden and Age (incl. Table S3 and Figure S2) .... p. 5

Zero-Order Correlations (Table S4) .................................................. p. 6

Robustness Analyses Results (Tables S5, S6, and S7) ......................... p. 7ff 


\section{Supplementary Figure S1: Growth Model}

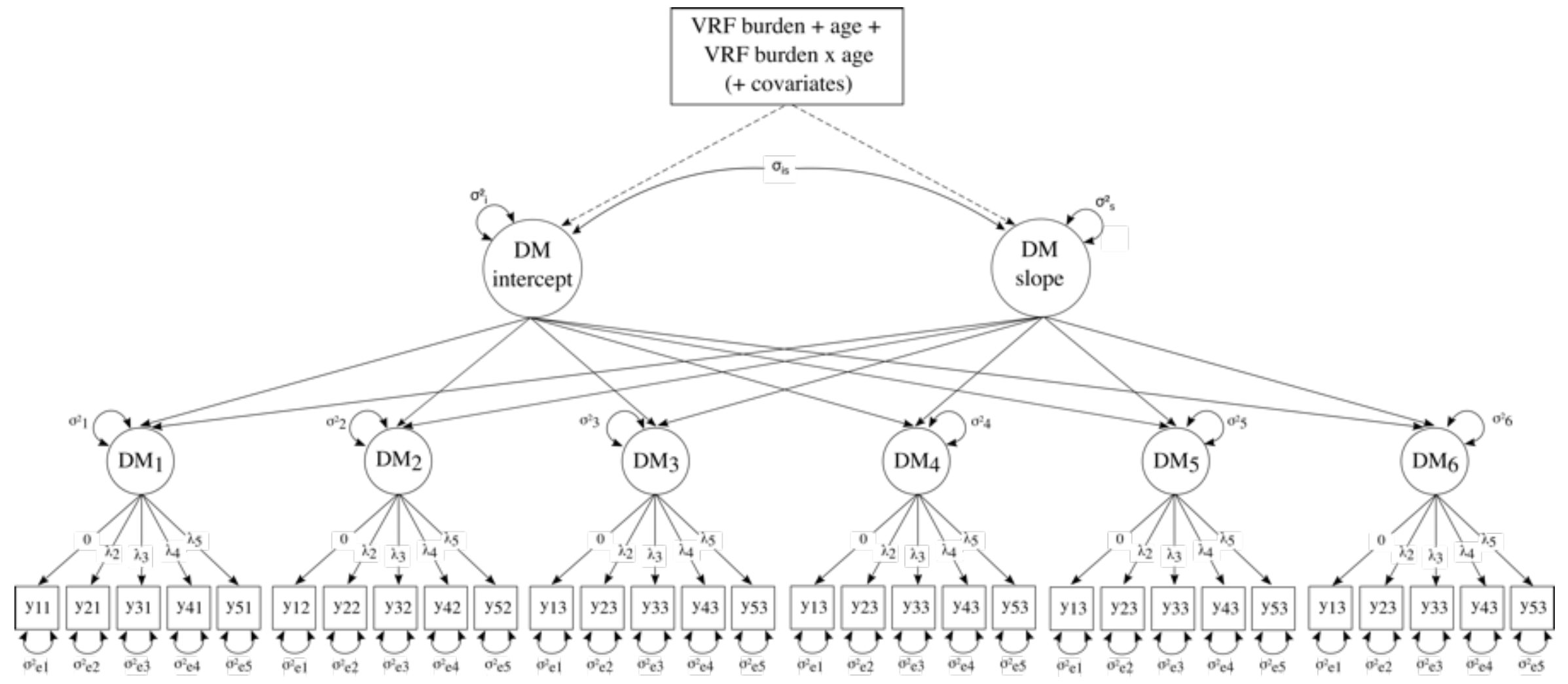

Figure 1. Illustration of the second-order latent growth model. The same latent construct for depressed mood (DM) was assessed using five items $\left(y_{\mathrm{ij}}\right)$ on each wave $\left(\mathrm{DM}_{1}, \mathrm{DM}_{2}, \ldots, \mathrm{DM}_{7}\right)$. A latent growth curve, consisting of initial levels (intercept; $\left.\mathrm{DM}_{\mathrm{i}}\right)$ and changes (slope; $\mathrm{DM}_{\mathrm{s}}$ ), was estimated across waves to describe a linear trajectory of depressed mood over time. The main parameters of interest - the association of vascular risk factor burden (VRF burden) and the interaction term between VRF burden and age with levels and changes in depressed mood are indicated by dashed lines. Although not shown for simplicity, correlations between the error terms of items across time (e.g. $y_{11}, y_{12}$, and $\left.y_{13}\right)$ were also modelled. 


\section{Supplementary Results 1: Measurement Invariance}

We examined longitudinal measurement invariance in the case of categorical indicators. In brief, a series of three confirmatory factor analyses (CFA) models assessing consecutive stages of measurement invariance was evaluated (see Liu et al. 2017):

- Model 1: First, a baseline model was fitted, testing the hypothesis that the same general pattern of factor loadings holds across all time points.

- Model 2: Second, a loading invariance model was fitted by additionally restraining the factor loadings to be qual across time.

- Model 3: Third, a unique factor invariance model was fitted by additionally constraining all residual variance terms to be equal over time.

Note that in all three models, the thresholds of the items were constrained to be equal across time and we furthermore allowed the residuals of the same items to be correlated across time. Models were estimated using a diagonally weighted least squares (DWLS) estimator with a mean and variance adjusted $\chi^{2}$ test statistic and robust standard errors (denoted WLSMV in the 'lavaan' package). We considered the highest level of MI to hold if (1) all models showed good fit to the data, and (2) the last model, which indicates unique factor invariance, did not show considerably worse fit than the preceding models. Good model fit was evaluated using several descriptive fit indices using the following cut-off values: $\mathrm{CFI} \geq .95, \mathrm{RMSEA}<.08$, SRMR $<$ .08. We also report results of global model fit tests and model comparisons, although results of the $\chi^{2}$ tests are likely to reject reasonable models given the large size sample size.

As can be seen in Table 2, all three models showed good model fit according to descriptive fit indices. Thus, we assumed the highest level of MI to hold. Factor loadings and residual variance terms for each of the three models across all waves are reported in Table S2.

Table S1

Results of Measurement Invariance Analysis.

\begin{tabular}{|c|c|c|c|c|c|c|c|c|c|}
\hline & \multicolumn{6}{|c|}{ Model fit } & \multicolumn{3}{|c|}{ Model comparison } \\
\hline & $\chi^{2}$ & $\mathrm{df}$ & $p$ & CFI & RMSEA & SRMR & $\Delta \chi^{2}$ & $\Delta \mathrm{df}$ & $p$ \\
\hline Model 1 & 989.2 & 315 & $<0.001$ & 0.983 & 0.022 & 0.042 & & & \\
\hline Model 2 & 1012.7 & 335 & $<0.001$ & 0.984 & 0.021 & 0.042 & 23.1 & 20 & 0.284 \\
\hline Model 3 & 1070.8 & 355 & $<0.001$ & 0.986 & 0.020 & 0.044 & 34.0 & 20 & 0.026 \\
\hline
\end{tabular}

Note: $N=5,689$; CFI $=$ Comparative Fit Index; RMSEA $=$ Root Mean Square Error of Approximation; SRMR = Standardised Root Mean Square Residual. 
Table S2

Unstandardised Factor Loadings $(\lambda)$ and Residual Variance Terms $\left(\sigma_{\varepsilon}^{2}\right)$ for the Three Measurement Invariance Models

\begin{tabular}{|c|c|c|c|c|c|c|c|c|c|c|c|c|}
\hline & \multicolumn{2}{|c|}{ Wave 2} & \multicolumn{2}{|c|}{ Wave 3} & \multicolumn{2}{|c|}{ Wave 4} & \multicolumn{2}{|c|}{ Wave 5} & \multicolumn{2}{|c|}{ Wave 6} & \multicolumn{2}{|c|}{ Wave 7} \\
\hline & $\lambda$ & $\sigma_{\varepsilon}^{2}$ & $\lambda$ & $\sigma_{\varepsilon}^{2}$ & $\lambda$ & $\sigma_{\varepsilon}^{2}$ & $\lambda$ & $\sigma_{\varepsilon}^{2}$ & $\lambda$ & $\sigma_{\varepsilon}^{2}$ & $\lambda$ & $\sigma_{\varepsilon}^{2}$ \\
\hline \multicolumn{13}{|l|}{ Model 1} \\
\hline Item 1 (I felt depressed) & 1.00 & 1.00 & 1.00 & 1.00 & 1.00 & 1.00 & 1.00 & 1.00 & 1.00 & 1.00 & 1.00 & 1.00 \\
\hline Item 2 (I was happy) & -1.10 & 1.00 & -1.15 & 1.31 & -1.17 & 1.13 & -1.27 & 0.77 & -1.25 & 1.14 & -1.26 & 1.32 \\
\hline Item 3 (I felt lonely) & 0.65 & 1.00 & 0.70 & 0.93 & 0.72 & 0.82 & 0.76 & 0.88 & 0.73 & 0.85 & 0.73 & 1.05 \\
\hline Item 4 (I enjoyed life) & -1.26 & 1.00 & -1.29 & 1.09 & -1.36 & 0.88 & -1.37 & 1.47 & -1.43 & 1.31 & -1.37 & 1.58 \\
\hline Item 5 (I felt sad) & 0.72 & 1.00 & 0.73 & 0.79 & 0.76 & 0.76 & 0.79 & 0.90 & 0.74 & 1.05 & 0.76 & 0.92 \\
\hline \multicolumn{13}{|l|}{ Model 2} \\
\hline Item 1 (I felt depressed) & 1.00 & 1.00 & 1.00 & 1.00 & 1.00 & 1.00 & 1.00 & 1.00 & 1.00 & 1.00 & 1.00 & 1.00 \\
\hline Item 2 (I was happy) & -1.21 & 1.00 & -1.21 & 1.36 & -1.21 & 1.16 & -1.21 & 0.82 & -1.21 & 1.24 & -1.21 & 1.40 \\
\hline Item 3 (I felt lonely) & 0.72 & 1.00 & 0.72 & 0.94 & 0.72 & 0.83 & 0.72 & 0.89 & 0.72 & 0.91 & 0.72 & 1.09 \\
\hline Item 4 (I enjoyed life) & -1.36 & 1.00 & -1.36 & 1.12 & -1.36 & 0.92 & -1.36 & 1.52 & -1.36 & 1.44 & -1.36 & 1.65 \\
\hline Item 5 (I felt sad) & 0.77 & 1.00 & 0.77 & 0.83 & 0.77 & 0.79 & 0.77 & 0.94 & 0.77 & 1.24 & 0.77 & 1.01 \\
\hline \multicolumn{13}{|l|}{ Model 3} \\
\hline Item 1 (I felt depressed) & 1.00 & 1.00 & 1.00 & 1.00 & 1.00 & 1.00 & 1.00 & 1.00 & 1.00 & 1.00 & 1.00 & 1.00 \\
\hline Item 2 (I was happy) & -1.14 & 1.00 & -1.14 & 1.00 & -1.14 & 1.00 & -1.14 & 1.00 & -1.14 & 1.00 & -1.14 & 1.00 \\
\hline Item 3 (I felt lonely) & 0.74 & 1.00 & 0.74 & 1.00 & 0.74 & 1.00 & 0.74 & 1.00 & 0.74 & 1.00 & 0.74 & 1.00 \\
\hline Item 4 (I enjoyed life) & -1.24 & 1.00 & -1.24 & 1.00 & -1.24 & 1.00 & -1.24 & 1.00 & -1.24 & 1.00 & -1.24 & 1.00 \\
\hline Item 5 (I felt sad) & 0.79 & 1.00 & 0.79 & 1.00 & 0.79 & 1.00 & 0.79 & 1.00 & 0.79 & 1.00 & 0.79 & 1.00 \\
\hline
\end{tabular}

Note: $N=5,689$. 


\section{Supplementary Results 2: Vascular Risk Factor Burden and Age}

Given the non-linear relationship between vascular risk factor (VRF) burden and age, we fitted a breakpoint regression model using the 'segmented package' in R (Muggeo, 2008). Breakpoint regression allows to estimate multiple linear trends in a data set, if there are a few values, i.e. breakpoints, where the effect of the predictors on the criterium changes abruptly (Muggeo, 2003).

Table S3

Results Breakpoint Regression Model Between Vascular Risk

Factor Burden and Age

\begin{tabular}{lll}
\hline Parameter & Estimate & SE \\
\hline Intercept & 0.873 & 0.142 \\
Slope left & 0.010 & 0.002 \\
Slope right & -0.057 & 0.010 \\
Breakpoint & 75.47 & 1.18 \\
\hline
\end{tabular}

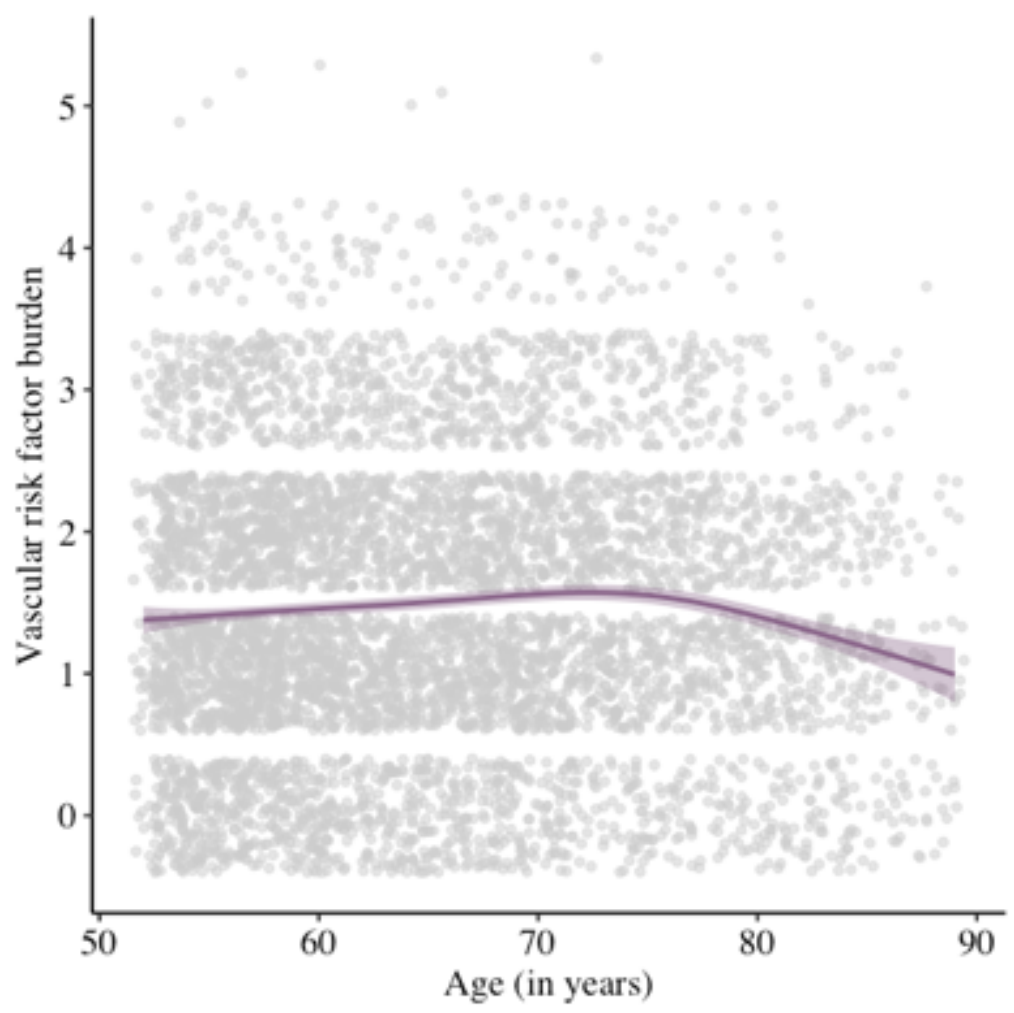

Figure S2. Non-linear relationship between age and vascular risk factor burden. 


\section{Supplementary Table S4: Zero-Order Correlations}

Table S4

Zero-Order Correlations

\begin{tabular}{|c|c|c|c|c|c|c|c|c|c|c|c|c|}
\hline & 1 & 2 & 3 & 4 & 10 & 11 & 12 & 13 & 14 & 15 & 16 & 17 \\
\hline 1. Age & - & & & & & & & & & & & \\
\hline 2. Gender & -0.04 & - & & & & & & & & & & \\
\hline 3. Ethnicity & -0.04 & 0.02 & - & & & & & & & & & \\
\hline 4. Education & -0.12 & 0.12 & 0.03 & - & & & & & & & & \\
\hline 10. VRF Burden & 0.00 & -0.04 & 0.02 & -0.09 & - & & & & & & & \\
\hline 11. ADL & 0.22 & -0.17 & 0.03 & -0.14 & 0.20 & - & & & & & & \\
\hline 12. Mood (W2) & 0.05 & -0.13 & 0.07 & -0.06 & 0.07 & 0.25 & - & & & & & \\
\hline 13. Mood (W3) & 0.07 & -0.11 & 0.05 & -0.08 & 0.08 & 0.25 & 0.48 & - & & & & \\
\hline 14. Mood (W4) & 0.07 & -0.13 & 0.03 & -0.06 & 0.11 & 0.26 & 0.43 & 0.45 & - & & & \\
\hline 15. Mood (W5) & 0.09 & -0.10 & 0.04 & -0.07 & 0.09 & 0.26 & 0.40 & 0.41 & 0.47 & - & & \\
\hline 16. Mood (W6) & 0.08 & -0.12 & 0.03 & -0.07 & 0.07 & 0.24 & 0.34 & 0.36 & 0.40 & 0.48 & - & \\
\hline 17. Mood (W7) & 0.10 & -0.10 & 0.05 & -0.07 & 0.09 & 0.21 & 0.35 & 0.36 & 0.41 & 0.44 & 0.49 & - \\
\hline
\end{tabular}

Note $: \mathrm{VRF}=$ vascular risk factor; $\mathrm{ADL}=$ activities of daily living; $\mathrm{W} 2=$ wave $2 ; \mathrm{W} 3=$ wave 3 ; $\mathrm{W} 4=$ wave 4 ; W5 $=$ wave 5 ; $\mathrm{W} 6=$ wave 6 


\section{Supplementary Results 3: Robustness Analyses}

Table S5

Robustness Analysis 1, Conditional First-Order Growth Curve Model $(N=5,689)$

\begin{tabular}{|c|c|c|c|c|c|c|}
\hline & \multicolumn{3}{|c|}{ Intercept } & \multicolumn{3}{|c|}{ Slope } \\
\hline & Est. & $95 \% \mathrm{CI}$ & Std. Est. & Est. & $95 \% \mathrm{CI}$ & Std. Est. \\
\hline \multicolumn{7}{|l|}{ Fixed effects ${ }^{a}$} \\
\hline & $-0.534 *$ & {$[0.489,0.579]$} & 0.625 & -0.008 & {$[-0.021,0.005]$} & -0.053 \\
\hline Age & -0.001 & {$[-0.004,0.003]$} & -0.004 & $0.003 *$ & {$[0.002,0.004]$} & 0.157 \\
\hline Gender $^{\mathrm{b}}$ & $-0.227^{*}$ & {$[-0.280,-0.174]$} & -0.132 & $0.017 *$ & {$[0.002,0.032]$} & 0.057 \\
\hline Education $^{\mathrm{c}}$ & -0.051 & {$[-0.119,0.018]$} & -0.021 & -0.002 & {$[-0.020,0.017]$} & -0.004 \\
\hline Ethnicity ${ }^{\mathrm{d}}$ & $0.543 *$ & {$[0.260,0.825]$} & 0.080 & -0.063 & {$[-0.133,0.008]$} & -0.054 \\
\hline ADL & $0.116^{*}$ & {$[0.100,0.131]$} & 0.315 & -0.001 & {$[-0.005,0.004]$} & -0.007 \\
\hline VRF burden & $0.040 *$ & {$[0.012,0.068]$} & 0.048 & -0.001 & {$[-0.009,0.008]$} & -0.007 \\
\hline VRF burden $\times$ age & $-0.004 *$ & {$[-0.007,-0.001]$} & -0.038 & 0.001 & {$[-0.001,0.001]$} & 0.025 \\
\hline \multicolumn{7}{|l|}{ Random effects } \\
\hline Variance & $0.620 *$ & {$[0.556,0.684]$} & 0.849 & $0.021 *$ & {$[0.016,0.025]$} & 0.969 \\
\hline Covariance & $-0.050^{*}$ & {$[-0.065,-0.035]$} & -0.440 & & & \\
\hline
\end{tabular}

\section{Model fit}

$\chi^{2}(\mathrm{df})$

$98.34(44)$

CFI

RMSEA

0.015

SRMR

0.015

Note. $\mathrm{CI}=$ confidence interval; $\mathrm{ADL}=$ activities of daily living; $\mathrm{VRF}=$ vascular risk factor

${ }^{\text {a }}$ The fixed effect now reflect sum scores of depressed mood; values therefore differ from the second-order growth model in the main analysis.

${ }^{\mathrm{b}}$ Reference category $=$ female

${ }^{\mathrm{c}}$ Reference category $=$ lower education

${ }^{\mathrm{d}}$ Reference category $=$ ' white'

$* p<.05$ 
Table S6

Robustness Analysis 2, Conditional First-Order Growth Curve Model $(N=6,835)$

\begin{tabular}{|c|c|c|c|c|c|c|}
\hline & \multicolumn{3}{|c|}{ Intercept } & \multicolumn{3}{|c|}{ Slope } \\
\hline & Est. & $95 \% \mathrm{CI}$ & Std. Est. & Est. & $95 \% \mathrm{CI}$ & Std. Est. \\
\hline \multicolumn{7}{|l|}{ Fixed effects ${ }^{\mathrm{a}}$} \\
\hline & $-0.525^{*}$ & {$[0.484,0.566]$} & 0.613 & -0.005 & {$[-0.017,0.007]$} & -0.036 \\
\hline Age & -0.001 & {$[-0.003,0.003]$} & -0.005 & $0.003 *$ & {$[0.002,0.003]$} & 0.161 \\
\hline Gender $^{\mathrm{b}}$ & $-0.200 *$ & {$[-0.249,-0.151]$} & -0.116 & 0.013 & {$[-0.001,0.026]$} & 0.043 \\
\hline Education $^{c}$ & -0.061 & {$[-0.127,0.006]$} & -0.024 & -0.001 & {$[-0.019,0.017]$} & -0.002 \\
\hline Ethnicity $^{\mathrm{d}}$ & $0.483^{*}$ & {$[0.253,0.713]$} & 0.074 & -0.048 & {$[-0.108,0.012]$} & -0.044 \\
\hline ADL & $0.115^{*}$ & {$[0.101,0.128]$} & 0.324 & -0.001 & {$[-0.005,0.003]$} & 0.013 \\
\hline VRF burden & $0.027 *$ & {$[0.001,0.053]$} & 0.032 & 0.002 & {$[-0.006,0.010]$} & -0.017 \\
\hline VRF burden $\times$ age & $-0.003 *$ & {$[-0.006,-0.001]$} & -0.036 & 0.001 & {$[-0.001,0.001]$} & 0.020 \\
\hline \multicolumn{7}{|l|}{ Random effects } \\
\hline Variance & $0.624^{*}$ & {$[0.565,0.682]$} & 0.851 & $0.020^{*}$ & {$[0.016,0.024]$} & 0.971 \\
\hline Covariance & $-0.047^{*}$ & {$[-0.060,-0.034]$} & -0.422 & & & \\
\hline \multicolumn{7}{|l|}{ Model fit } \\
\hline$\chi^{2}(\mathrm{df})$ & \multicolumn{6}{|c|}{$92.21(44)$} \\
\hline CFI & \multicolumn{6}{|c|}{0.992} \\
\hline RMSEA & \multicolumn{6}{|c|}{0.012} \\
\hline SRMR & \multicolumn{6}{|c|}{0.013} \\
\hline
\end{tabular}

Note. $\mathrm{CI}=$ confidence interval; $\mathrm{ADL}=$ activities of daily living; $\mathrm{VRF}=$ vascular risk factor

${ }^{a}$ The fixed effect now reflect sum scores of depressed mood; values therefore differ from the second-order growth model in the main analysis.

${ }^{\mathrm{b}}$ Reference category $=$ female

${ }^{\mathrm{c}}$ Reference category $=$ lower education

${ }^{\mathrm{d}}$ Reference category $=$ 'white'

$* p<.05$ 
Table S7

Robustness Analysis 3, Conditional First-Order Growth Curve Model with Sampling Weights $(N=5,689)$

\begin{tabular}{|c|c|c|c|c|c|c|}
\hline & \multicolumn{3}{|c|}{ Intercept } & \multicolumn{3}{|c|}{ Slope } \\
\hline & Est. & $95 \% \mathrm{CI}$ & Std. Est. & Est. & $95 \% \mathrm{CI}$ & Std. Est. \\
\hline \multicolumn{7}{|l|}{ Fixed effects ${ }^{\mathrm{a}}$} \\
\hline & $-0.531^{*}$ & {$[0.484,0.577]$} & 0.613 & -0.007 & {$[-0.020,0.006]$} & -0.049 \\
\hline Age & -0.001 & {$[-0.004,0.003]$} & -0.003 & $0.002 *$ & {$[0.001,0.003]$} & 0.157 \\
\hline Gender $^{\mathrm{b}}$ & $-0.213^{*}$ & {$[-0.268,-0.159]$} & -0.123 & 0.015 & {$[-0.001,0.030]$} & 0.051 \\
\hline Education $^{\mathrm{c}}$ & -0.050 & {$[-0.121,0.021]$} & -0.019 & 0.001 & {$[-0.017,0.020]$} & 0.003 \\
\hline Ethnicity $^{\mathrm{d}}$ & $0.554^{*}$ & {$[0.268,0.841]$} & 0.092 & -0.064 & {$[-0.138,0.011]$} & -0.063 \\
\hline $\mathrm{ADL}$ & $0.119^{*}$ & {$[0.103,0.135]$} & 0.320 & -0.001 & {$[-0.005,0.004]$} & -0.008 \\
\hline VRF burden & $0.043^{*}$ & {$[0.014,0.073]$} & 0.051 & -0.001 & {$[-0.009,0.008]$} & -0.004 \\
\hline VRF burden $\times$ age & $-0.004 *$ & {$[-0.007,-0.001]$} & -0.039 & 0.001 & {$[-0.001,0.001]$} & 0.027 \\
\hline \multicolumn{7}{|l|}{ Random effects } \\
\hline Variance & $0.634 *$ & {$[0.567,0.700]$} & 0.845 & $0.020^{*}$ & {$[0.016,0.025]$} & 0.969 \\
\hline Covariance & $-0.050^{*}$ & {$[-0.065,-0.035]$} & -0.422 & & & \\
\hline \multicolumn{7}{|l|}{ Model fit } \\
\hline$\chi^{2}(\mathrm{df})$ & \multicolumn{6}{|c|}{$92.40(44)$} \\
\hline CFI & \multicolumn{6}{|c|}{0.990} \\
\hline RMSEA & \multicolumn{6}{|c|}{0.014} \\
\hline SRMR & \multicolumn{6}{|c|}{0.015} \\
\hline
\end{tabular}

Note. $\mathrm{CI}=$ confidence interval; $\mathrm{ADL}=$ activities of daily living; $\mathrm{VRF}=$ vascular risk factor

${ }^{\text {a }}$ The fixed effect now reflect sum scores of depressed mood; values therefore differ from the second-order growth model in the main analysis.

${ }^{\mathrm{b}}$ Reference category $=$ female

${ }^{\mathrm{c}}$ Reference category $=$ lower education

${ }^{\mathrm{d}}$ Reference category $=$ 'white'

$* p<.05$ 


\section{Supplementary References}

Liu, Y., Millsap, R. E., West, S. G., Tein, J.-Y., Tanaka, R., \& Grimm, K. J. (2017). Testing measurement invariance in longitudinal data with ordered-categorical measures. Psychological Methods, 22(3), 486-506. https://doi.org/10.1037/met0000075

Muggeo, V. M. R. (2003). Estimating regression models with unknown break-points. Statistics in Medicine, 22(19), 3055-3071. https://doi.org/10.1002/sim.1545

Muggeo, V. M. R. (2008). Segmented: An R Package to Fit Regression Models With Broken-Line Relationships. $R$ News, 8, 20-25. 